\title{
802 ヘルスケアや環境保全のためのリング型 PZT 薄膜微小共振子の研究開発 Development of A Ring-shaped PZT Film Resonator for Health Care and Environmental Applications
}

\author{
李 暁強（茨城大） 1 \\ ○ 佐川 貴久（茨城大）' \\ 正王 東方 (茨城大) ${ }^{1, *}$ 魯 健 (産総研) ${ }^{2}$ 前田 龍太郎 (産総研) ${ }^{2}$ \\ Li Xiaoqiang ', O Takahisa Sagawa ', Dong F. Wang ', Lu jian ${ }^{2}$, and Ryutaro Maeda ${ }^{2}$
}

\footnotetext{
' Micro Engineering \& Micro Systems Laboratory, Ibaraki University (College of Eng.), Hitachi, Ibaraki 316-8511, Japan (Tel: +81-294-38-5024; Fax: +81-294-38-5047; E-mail: dfwang@mx.ibaraki.ac.jp)

${ }^{2}$ Research Center for Ubiquitous MEMS and Micro Engineering (UMEMSME), AIST, Tsukuba, Ibaraki 305-8564, Japan
}

\begin{abstract}
The eigenstate shift in resonant frequency by a ring-shaped resonator has been studied for ultrasensitive mass detection, in liquid environment. The proposed ring-shaped resonator is comprised with a multilayer of $\mathrm{Pt} / \mathrm{Ti} / \mathrm{PZT} / \mathrm{Pt} / \mathrm{Ti} / \mathrm{SiO}_{2}$ deposited on a silicon-on-insulator (SOI) wafer and is expected to be excited in a contour mode. In order to estimate the sensitivity of the ring-shaped resonator to small mass perturbation, theoretical analysis has been conducted for eigenstate shifts with or without small perturbation, from the view point of geometrical design and the mass impression methodology.
\end{abstract}

Key words: Ring-shaped resonator, Eigenstate shift, Structural design, Micro-fabrication, PZT thin film, Mass perturbation

\section{Introduction}

The term biosensor is an analytical device which could converts biological responses into electrical signals [1]. In recent years, the film bulk acoustic resonator (FBAR) had been attracted increasing considerations in the field of microwave circuits and finds its potential for biosensor application. The physical miniaturization of FBAR allows it could be fabricated in batch by Micro Electro Mechanical Systems (MEMS) technology. Generally, FBAR typically consists of a sandwich structure in which a suspended piezoelectric thin film of $\mathrm{ZnO}$ or aluminium nitride (AIN) by two metal electrodes. The thin film of piezoelectric materials allows the ring-shaped biosensor to response a high resonant frequency (up to $10 \mathrm{GHz}$ ) and also the large frequency sensitivity to a mass loading $\left(>1000 \mathrm{~Hz} \mathrm{~cm}^{2}\right.$, $\mathrm{ng}$ ).

C-reactive proteins (CRP) are found in the blood, the level rising of which in response to certain tissue inflammation (i.e. C-reactive protein is an acute-phase protein) as well as rapid angiogenesis of tumors. Therefore, it is believed that effective and sensitive detection of CPR levels in blood has great potential in the application of healthcare field.

In Xu et al.'s study [2], an acoustic resonator of high quality factors (Qs) was reported. Ring-shaped piezoelectric AlN thin film was made as FBAR. The resonator was characterized by an aptamer-thrombin binding pair for a biosensor and showed a mass resolution of $1.78 \mathrm{ng} / \mathrm{cm}^{2}$. In this work however, we study the eigenstate shift in resonant frequency due to a mass perturbation in a proposed ring-shaped PZT film resonator, and shows a rapid and competitive sensitivity, apart from the miniaturization of resonator device itself.

\section{Design of ring-shaped PZT film resonator}

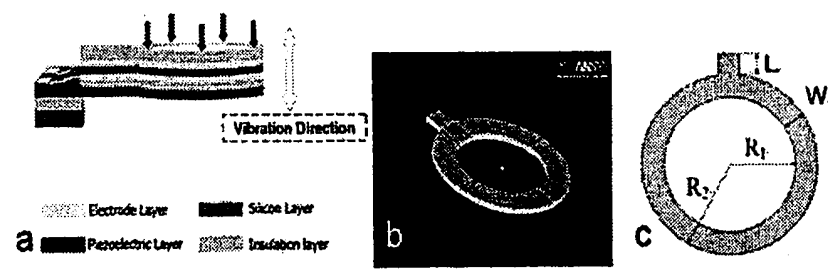

Figure 1: a) The proposed ring-shaped PZT film resonator for bio-sensing applications in a liquid environment; $b$ ): The model for theoretical analyses by using commercial ANSYS software; c): The definitions of length $L$, width $W, R_{l}$, and $R_{2}$, in geometrical design.
Fig. 1(a) is the basic model of ring-shaped PZT film resonator resonator is consisted of $\mathrm{SiO}_{2}, \mathrm{Si}, \mathrm{PZT}, \mathrm{Pt}$ and $\mathrm{Si}$ layers. PZT film generate electric signals. Both sides of PZT film, Pt layers were used as electrode to collect and transfer the electric signal from PZT film A silicon substrate with thickness of $2 \mu \mathrm{m}$ was used to support all the films of electrodes and PZT. The model for theoretical analyses and the definitions of geometrical sizes are shown in Fig. 1(b) and (c), respectively. The ring has the inner and outer diameter of $R_{1}$ and $R_{2}(\leq 100 \mu \mathrm{m})$. Therefore, the width of the ring could be described as $W=R_{2}-R_{1}$. There is also a support which is fixed and the length of this support is described as $L$. Obviously, the frequency shift of the ring-shaped resonator is affected by both factors of $L$ and $W$. In order to estimate the sensitivity to small mass perturbation of the eigenstate (resonant frequency) of the ring-shaped resonator, theoretical analysis has been conducted for eigenstate shifts with and without small perturbation using ANSYS software.

\section{Simulation results and discussion}
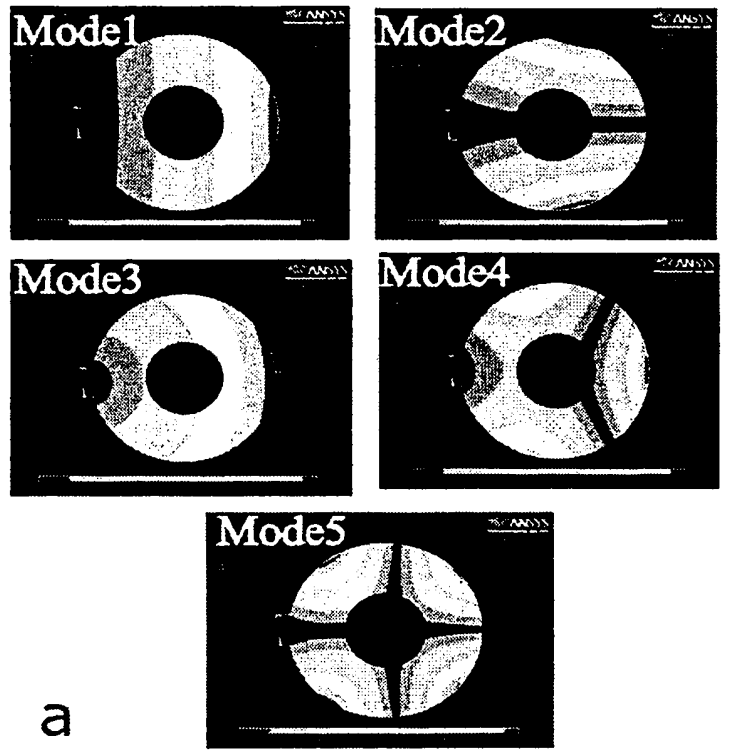

a

Figure 2: a) Frequency shift as a function of length, with a relation to different vibration modes. 

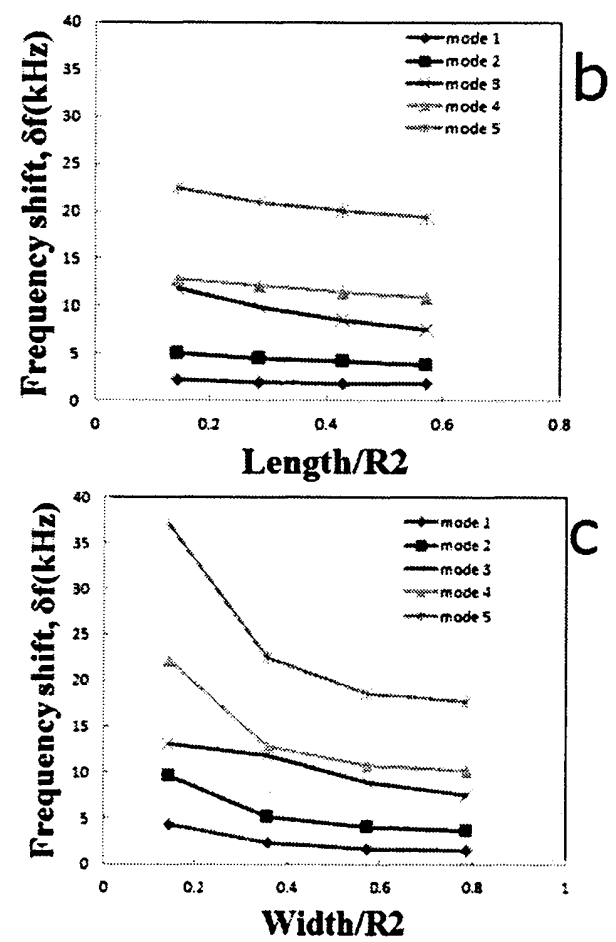

Figure 2: b) Frequency shift as a function of length, with a relation to different vibration modes; and c) Frequency shift as a function of width.

In order to examine the possibility of testing the small mass perturbation by the ring-shaped resonator, 10 and $20 \mathrm{ng}$ water were assumed put on the top of this ring-shaped resonator, thereafter, a driven force was supplied and then the data of frequency shift was obtained by ANSYS results. That is, the obvious frequency shifts result in great significance in testing of the small mass perturbation.

There are various frequency behaviours existing on the ring-shape resonator, however, 5 different and typical frequency modes were discussed in this work. As shown in Fig. 2 b), there are the eigenstate shifts in vibration Mode 1 to Mode 5 (shown in Fig. 2 a) as a function of length, before and after a mass perturbation of $10 \mathrm{ng}$ was induced. With the increasing of the ring-handle length, the frequency shift presents a downtrend in the data-graph. Especially on Mode 3, the frequency shift dropped about $5 \mathrm{kHz}$ when the length of ring-shaped resonator changed from 0.14 to 0.56 of Length/ $R_{2}$. On the vibration Mode 1 and 2, the frequency shifts were mostly the same as the length changed. Fig. $2 \mathrm{c}$ ) shows eigenstate shifts in vibration Mode 1 to Mode 5 as a function of width, before and after a mass perturbation $10 \mathrm{ng}$ was induced. Unlike the affection of handle length, with the increasing of ring width, all of the 5 modes had the significant downtrends. The most obvious downtrend was present in Mode 5 that the frequency shift decreased from $37 \mathrm{kHz}$ to $18 \mathrm{kHz}$ when the width of ring-shaped resonator increased from 0.14 to 0.78 of width $/ \mathbf{R}_{2}$.

The frequency shifts from 2.29 to $4.33 \mathrm{kHz}$ can then be obtained when excited in the contour mode. Fig. $3 \mathrm{a}$ and $\mathrm{b}$ ) show the frequency shifts in vibration Mode 3 and Mode 5, respectively, due to the mass perturbation as a function of $R_{1}$ as defined in Fig. $1 \mathrm{c})$. The monotonous behaviour of frequency shift in Mode 5 can be qualitatively explained by Equation (1), where $R_{1}$ and $R_{2}$ are the inner and outer radii of the ring, and $E$ and $\rho$ are the Young's modulus and density of silicon respectively. However, the extreme point is derived for optimum design as observed in Figure 3 b).

$$
f_{0}=\frac{1}{2 \pi \sqrt{R_{1} R_{2}}} \sqrt{\frac{E}{\rho}}
$$
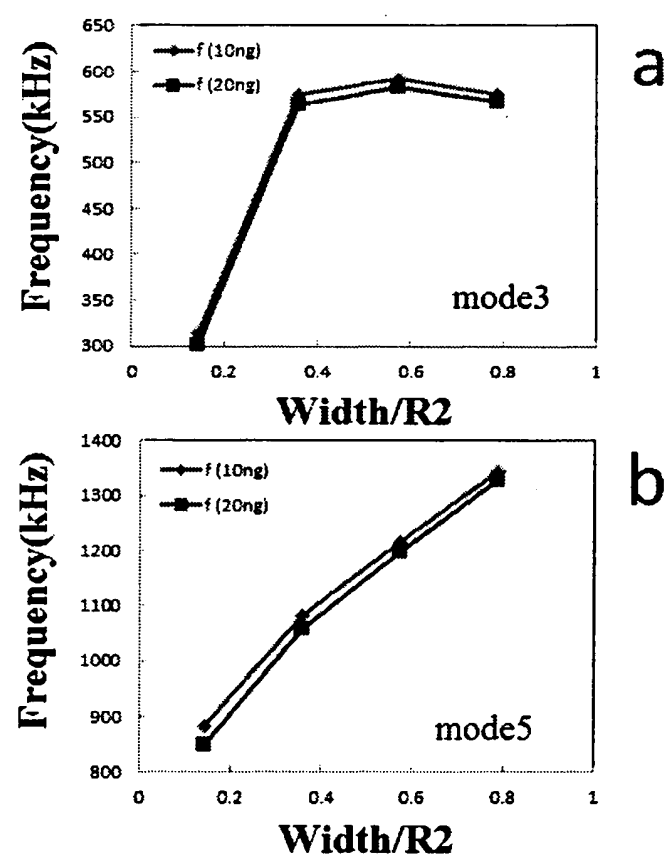

Figure 3: Frequency shift as a function of $R_{1}$ ( $a$ : Mode 3; $b$ : Mode $5)$.

\section{Future prospects}

Based on the above results of both vibration modes and relevant frequency shifts due to the small mass perturbation, a novel design with a water droplet, as shown in Fig. 4, has been thus proposed for further consideration.

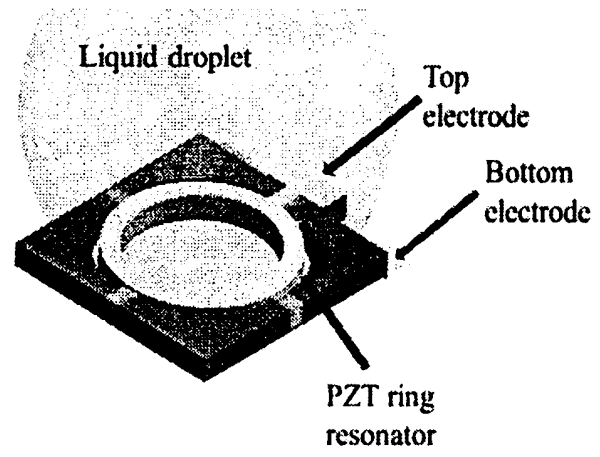

Figure 4: Sketch of the proposed ring-shape resonator.

\section{Conclusions}

The novel design of the ring-shaped resonator based on PZT film was made and the resonator was aimed to manufacture biosensor which could be utilized in liquid environment.

The capability of the resonator that responding to small mass perturbation of liquid media was simulated and tested by ANSYS software, based on various parameters of ring-handle lengths and ring widths. The results indicated that when a mass perturbation (i.e., a liquid droplet) of $10 \mathrm{ng}$ was homogeneously contacting on the top insulation layer of the resonator, frequency shifts at least from 2.29 to $4.33 \mathrm{kHz}$ could be obtained.

\section{References}

[1] Yan Z, Zhou X, Pang G, Zhang T, Liu W, Cheng J, Song Z, Feng S, Lai L, Chen J, and Wang Y. ZnO-based film bulk acoustic resonator for high sensitivity biosensor applications. Appl Phys Lett 2007; 90: 143503-5.

[2] Xu W, Choi S, and Chae J. A contour-mode film bulk acoustic resonator of high quality factor in a liquid environment for biosensing applications. 2010; 96: 053703-053705. 